\title{
Idiopathic Spinal Cord Herniation at Thoracic Spine Level: A Case Report
}

\author{
Tae Woong Bae, Woojoo Lee, Young Jin Kim \\ Department of Neurosurgery, Dankook University College of Medicine, Cheonan, Republic of Korea
}

Corresponding author:

Young Jin Kim $\mathrm{MD}, \mathrm{PhD}$

Department of Neurosurgery,

Dankook University Hospital, 201

Manghyang-ro, Dongnam-gu,

Cheonan 31116, Republic of Korea

Tel: +82-2-2231-1591

E-mail: spine1225@naver.com

Received: March 30, 2021

Revised: April 17, 2021

Accepted: April 19, 2021

\begin{abstract}
Idiopathic spinal cord herniation (ISCH) is a rare disease. It especially occurs at the thoracic level through a ventral dural defect without a definite pathophysiology. More patients have been diagnosed recently because both technical quality and accessibility to magnetic resonance image have increased. Symptoms of ISCH are related to anterior cord syndrome due to the location of cord herniation in the ventral portion. Progressive myelopathy and complete or partial Brown-Sequard syndrome are also commonly observed. Spinal cord reposition or dural defect repair are treatments of choice. We present a case of ISCH at T4-5 level of a patient whose symptom of chronic back pain turned into Brown-Sequard syndrome. It was managed with surgical treatment, spinal cord reposition, and dural defect repair with an artificial dura patch. En bloc laminoplasty was also performed to prevent deformity and CSF leakage.
\end{abstract}

Key Words: Herniation, Spinal cord, Brown-Sequard syndrome, Thoracic spine

\section{INTRODUCTION}

Herniation of the spinal cord specifically presents at the thoracic level through a ventral dural defect without a definite pathophysio$\log y^{1,5-7}$. Since its initial description by Wortzman et al. in 1974, more patients have been diagnosed recently because both technical quality and accessibility to magnetic resonance imaging (MRI) have increased ${ }^{3,7,12}$. Its clinical symptoms show with progressive myelopathy including Brown-Sequard syndrome because the location of the cord hemiation is at the ventral portion. About two thirds of cases occur in female patients with an insidious symptom onset ${ }^{3}$. Spinal cord reposition and dural defect repair with an artificial dura are the best surgical options.

We introduce a case of idiopathic spinal cord herniation (ISCH) at T4-5 level successfully managed with surgical treatment, spinal cord reposition, and dural defect repair. After reposition was done, en bloc laminoplasty at the T4-5 level was also performed to prevent cerebrospinal fluid (CSF) leakage with compression and preserve the function of muscles to reattach at the lamina, thus protecting from the outside environment after recovery from surgery.

\section{CASE REPORT}

A 42-year-old male without any underlying disease suffered from a progressive motor weakness and paresthesia. The patient complained about his deteriorated symptoms from five months. These deteriorated signs were progressive paresthesia, loss of temperature, sensation for pain in his left lower extremities, and decreased motor grade on his right side. In physical examination, the patient's motor grade was $4 \sim 4+$ on both side which makes the patients hard to ambulate. Decreased right foot dorsiflexion with a grade of about 3 was also observed. Electromyography performed for intraoperative monitoring (IOM) before surgical treatment suggested a central conduction defect on both lower extremities. A cervicothoracic junction MRI revealed ISCH at the T4-5 level and CSF collection at dilatated subarachnoid space. Both MRI and CT myelography were performed after admission for evaluation (Figure 1-2). Under general anesthesia, neuromonitoring with MEPs and SEPs was put in place and the patient was turned into a prone position. After identification of the thoracic level with a C-arm, total laminectomy was performed with a bone scalpel at T4 and T5 levels. Under the microscope, the dura was opened sagittally. After cutting ipsilateral dentate ligaments and

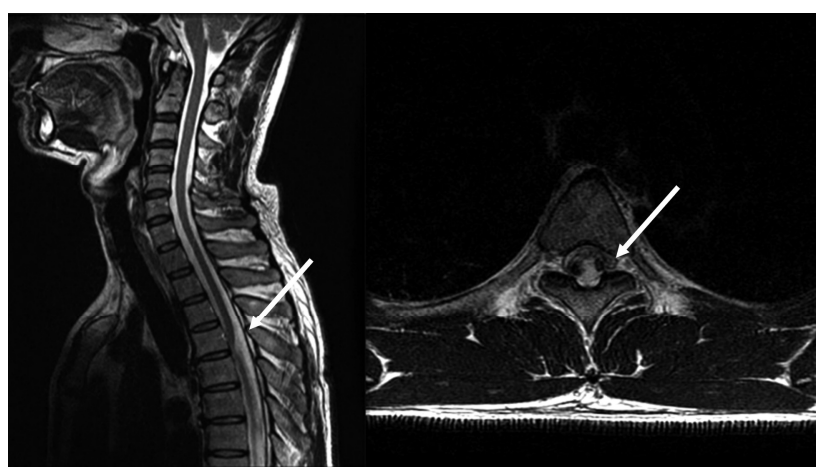

Figure 1. Imaging features of ISCH. MRI T2 sequence showed fluid collection at dorsal portion of the T4-5 level with a sharp angulation of the thoracic cord suggesting ventral protrusion of spinal cord. 
carefully pushing spinal cord towards the contralateral side, spinal cord hemiation and dural defect were visible through the microscope (Figure 3). Oval shaped about $2 \mathrm{~cm}$ size dural defect and cord herniation without inflammation or adhesion was observed at the ventral portion of spinal cord. The margin was soft and there was no sign of spinal cord swelling. Using a micro-dissector, dural defect was explored and reposition of the herniated spinal cord was performed carefully. An artificial dura was overlapped intradurally to cover

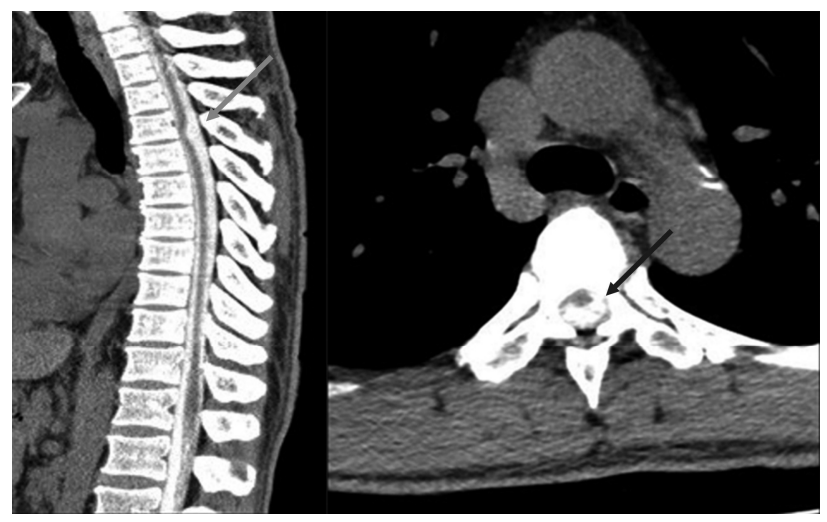

Figure 2. Sagittal and axial CT myelogram images demonstrating sharp angulation of the thoracic cord, CSF fluid collection, and widening of the dorsal subarachnoid space.

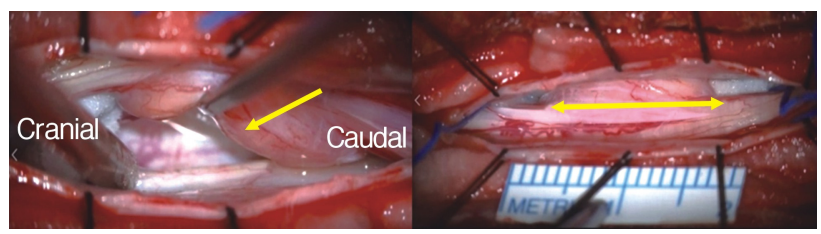

Figure 3. Intraoperative view under a microscope. Herniation of spinal cord and dural defect are also observed. Oval shaped $2 \mathrm{~cm}$ size dural defect and herniation was observed and there was no sign of adhesion or inflammation and spinal cord swelling.

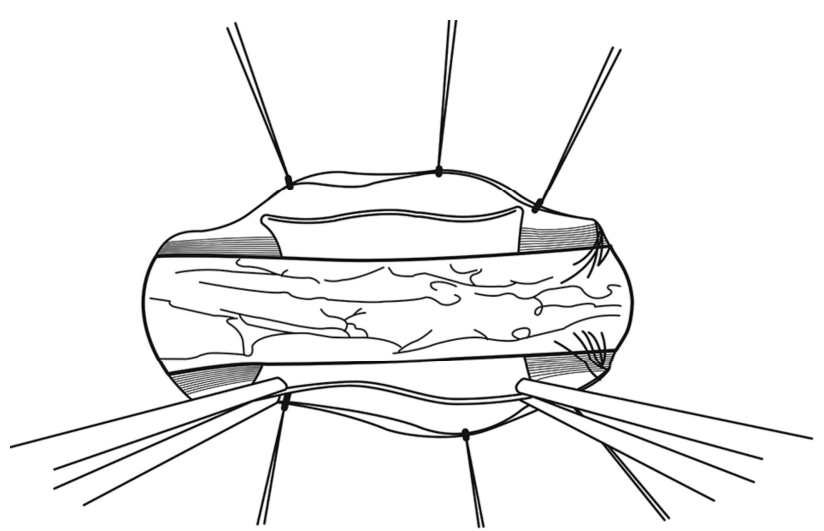

Figure 4. Simple pictures for intraoperative view while overlapping the dura. The dura was wrapped with artificial dura. After wrapping, the dura was tightly sutured with continuous suture to prevention recurrence. the dural defect (Figure 4). Due to the size of dural defect, we decided to overlapping the defect with an artificial dura instead of suture. After overlapping, the dura incision was closed tightly with a continuous suture. No MEP or SEP alteration was noted during the entire procedure. Presenving the muscle's function by reattaching the muscle to the reinserted bone after surgery for protection from external injury was considered (Figure 5).

Symptoms were improved after surgery. Paresthesia and motor weakness were improved and the patient was discharged at 7 days after the surgery. When the patient came to the hospital after discharge, there was no neurologic deficit postoperatively. MR image at the follow up showed no recurrence of spinal cord herniation (Figure 6).

\section{DISCUSSION}

Although $\mathrm{ISCH}$ is a rare disease, its diagnosis and treatment are important because it is one of spinal cord lesions that could be cured with surgical repositioning and dura repair. Diagnosis is usually done with MRI. Computed tomography-myelography can also be used based on radiographic features such as ventral herniation of the spinal cord, abnormal dilatation of the subarachnoid space, and contracture or torsion of the spinal cord'.

Most clinical manifestations that can be observed in other cases are ipsilateral hemiparesis with sensory loss and vibration, loss of pain and temperature at the contralateral side, and Brown-Sequard syndrome. Some cases of bilateral motor weakness and bowel and bladder dysfunction have been reported'. In the present case, the patient's initial clinical manifestation was a loss of sensory on the left side due to dysfunction of the lateral spinothalamic tract and progressive spinal cord herniation induced motor weakness of lower extremities. However, proprioception and vibration were relatively intact because the posterior portion of the cord was affected after progression of the spinal cord herniation.

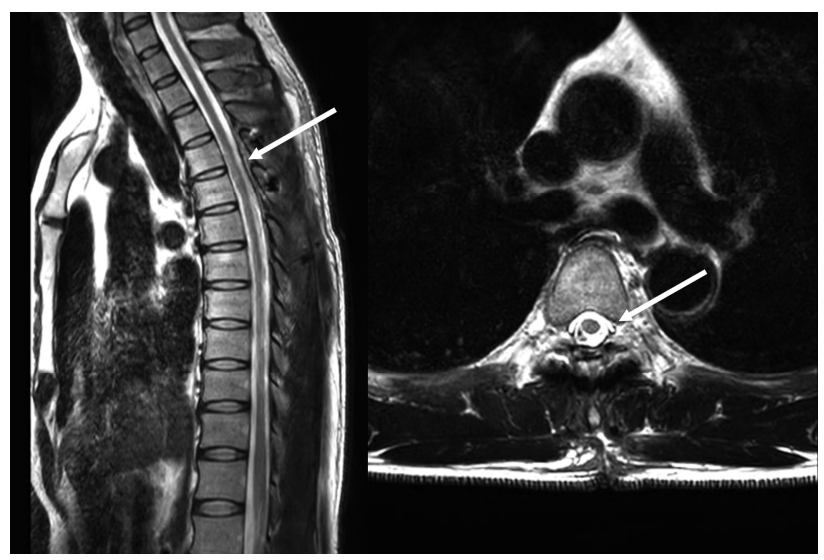

Figure 5. Follow-up MRI image after recovery from surgery. There was no recurrence of spinal cord herniation. Resolution of fluid collection at the dorsal portion of spinal cord due to dilatation of subarachnoid space is also observed. 
The pathophysiology of idiopathic spinal cord herniation remains unclear. A few theories have been suggested. In these theories, the critical point is how dural defects are formed. A history of trauma may be the reason for idiopathic spinal cord herniation. This was first reported by Tekkok ${ }^{13}$. The author explained that a traumatic event 35 years ago might not have a large impact on the dural defect.

Repetition of minor trauma might cause idiopathic spinal cord herniation ${ }^{3}$. However, it has not been reported yet. Most cases of idiopathic spinal cord herniation are related to subarachnoid cysts because abnormal dilatation of the subarachnoid space is considered to be the result of cord herniation. Erosion of the dura due to compression has been reported as a cause of $\mathrm{ISCH}^{2,8}$. Another theory is a congenital defect of meninges. However, all cases reported in the past did not detect any spinal anomaly at other spine levels. Duplication of meninges in the inner layer at the ventral portion causing herniation and incarceration is also another theory ${ }^{11}$, but there is no affirmation of radiological or pathophysiological evidence.

Progression of the spinal cord herniation is sustained after the cord is placed on the dural defect point and adhesion is started ${ }^{4}$. Due to connecting of the subarachnoid and epidural space, CSF can move into the cyst by pulsation. When adhesion between the defect and cord is completed, pulsatile of CSF can push the cord out of the dura, resulting in spinal cord herniation ${ }^{10,13}$. Although several theories exist, its exact pathophysiology is still unknown which makes this patient's cause of cord herniation uncertain. But we anticipated that repeated minor trauma which the patient did not recognize might cause cord herniation because there was no sign of inflammation and specific medical or trauma history.

Thoracic spinal cord herniation has a couple common characteristics, including ventral herniation at levels T2-8 and subarachnoid cysts. Although the reason for the prevalence of idiopathic spinal cord herniation at the thoracic level is still an ongoing debate, its common physiologic characteristics are thought to be kyphosis and limited mobility compared to cenvical and lumbar levels ${ }^{13}$.

The treatment of choice for an idiopathic spinal cord herniation includes repositioning of the cord and repair of the dural defect with surgery. When repairing the dural defect, we used an artificial dura to overlap the defective area. However, depending on the size and shape, the dural defect may be sutured without overlapping. Also, recovered neurologic deficit was observed after surgery.

\section{CONCLUSION}

ISCH is not a common disease. With improving quality of imaging tools, it should be considered more for progressive myelopathy patients. Although progressive severe myelopathy might occur, it can be treated with surgical repositioning. Laminoplasty following a cord reposition and dura repair seem to be promising and helpful for preventing
CSF leakage and potential deformity, protecting from environmental forces, and insulating against muscle hypotrophy following laminectomy.

\section{REFERENCES}

1. Bhatia K, Madhavan A, Coutinho C \& Mathur S: Idiopathic spinal cord herniation. Clin Radiol 75:721-729, 2020

2. Borges LF, Zervas NT \& Lehrich JR: Idiopathic spinal cord herniation: A treatable cause of the Brown-Sequard syndromeCase report. Neurosurgery 36:1028-1032; discussion 1032-1033, 1995

3. Carter BJ, Griffith BD, Schultz LR, Abdulhak MM, Newman DS \& Jain R: Idiopathic spinal cord herniation: An imaging diagnosis with a significant delay. Spine J 15:1943-8, 2015

4. Dix JE, Griffitt W, Yates C \& Johnson B: Spontaneous thoracic spinal cord herniation through an anterior dural defect. AJNR Am J Neuroradiol 19:1345-1348, 1998

5. Ghali MGZ, Srinivasan VM, Rao VY \& Omeis I: Idiopathic thoracic spinal cord herniation. J Clin Neurosci 51:1-5, 2018.

6. Haber MD, Nguyen DD \& Li S: Differentiation of idiopathic spinal cord herniation from CSF-isointense intraspinal extramedullary lesions displacing the cord. Radiographics 34:313329, 2014

7. Imagama S, Matsuyama Y, Sakai Y, Nakamura H, Katayama Y, Ito Z, Wakao N, Sato K, Kamiya M, Kato F, Yukawa Y, Miura Y, Yoshihara H, Suzuki K, Ando K, Hirano K, Tauchi $\mathrm{R}$, Muramoto A \& Ishiguro N: Image classification of idiopathic spinal cord herniation based on symptom severity and surgical outcome: A multicenter study. J Neurosurg Spine 11:310319, 2009

8. Isu T, lizuka T, Iwasaki Y, Nagashima M, Akino M \& Abe H: Spinal cord herniation associated with an intradural spinal arachnoid cyst diagnosed by magnetic resonance imaging. Neurosurgery 29:137-139, 1991

9. Kumar R, Taha J \& Greiner AL: Herniation of the spinal cord. Case report. J Neurosurg 82:131-136, 1995

10. Nakazawa H, Toyama Y, Satomi K, Fujimura Y \& Hirabayashi $\mathrm{K}$ : Idiopathic spinal cord herniation. Report of two cases and review of the literature. Spine (Phila Pa 1976) 18:2138-141, 1993

11. Sasani M, Ozer AF, Vural M \& Sarioglu AC: Idiopathic spinal cord herniation: Case report and review of the literature. $\mathrm{J}$ Spinal Cord Med 32:86-94, 2009

12. Tekkök IH. Spontaneous spinal cord herniation: Case report and review of the literature. Neurosurgery 46:485-491; discussion 491-492, 2000

13. Wortzman G, Tasker RR, Rewcastle NB, Richardson JC \& Pearson FG: Spontaneous incarcerated herniation of the spinal cord into a vertebral body: A unique cause of paraplegia. Case report. J Neurosurg 41:631-635, 1974 